\title{
Real-time Traffic Jam Detection from UAV Using Cascade Haar
}

\author{
[Saad B. Alotaibi, Amer S. Alharthi]
}

\begin{abstract}
This paper contains an in depth work carried out in the area of real-time vehicle detection from an Unmanned Ariel Vehicle (UAV) to detect the traffic jam by counts the number of cars in the roadway. The optical camera located inside the UAV takes images from a particular angle and analysis is performed on the image to detect the vehicle (static or moving). To obtain the accurate and quick result, the analysis should be in ground station. This paper presents a highly reliable technique to detect many different types of vehicles from images having variant backgrounds. This technique used Haar classifiers to perform this real-time application. The Haar classifier was trained with multiple features to accomplish the task. The task was successfully achieved with minimum false positive rate.
\end{abstract}

Keywords-UAV, Haar Classifier, vehicle Detection

\section{Introduction}

Real time detection of vehicles from aerial view is particularly useful for surveillance and other monitoring activities. Usage of UAV (cf. figure 1) with a fitted camera is an excellent option to carry out this activity. In addition, to speed up the computational process we send the video frames (images) to ground station for analysing and processing. To send video frames we used a high quality communication system (cf. figure 2) to send images from UAV and receive it in ground station. However, one should have an appropriate method/algorithm to detect the vehicle presence from the acquired image. Above all, the algorithm must be capable to detect the object with different kinds of background and environmental conditions. The ratio of the object to the image as taken from the UAV will be very small. Objects such as humans and cars are highly dynamic and available in variety of shape, colour and size. Detection of a dynamic object with variant backgrounds under the above-mentioned conditions in real time is a highly challenging task. In this paper, we present a suitable solution to the mentioned task.

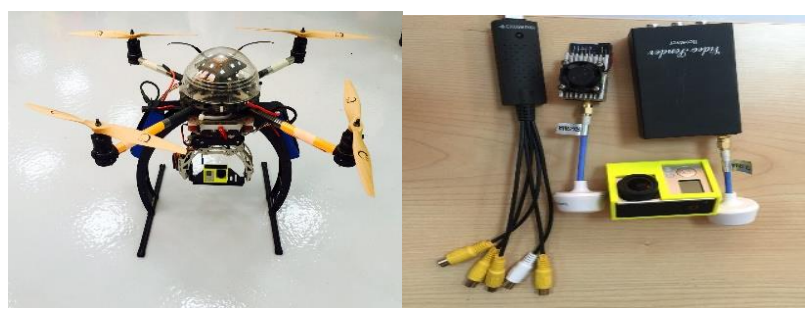

Figure 1 : UAV

Figure 2 : Com. System

Haar Cascades classifier is one of the recognized algorithms for object detection in an image with minimal computer processing. The Haar Cascade algorithm has a reasonable accuracy for detecting even dynamic objects (same object with multiple forms) such as human face. The commonly available algorithm reads the pixel color and plays with its intensity values to detect objects. In contrary to common algorithms, Haar Cascade classifier regionalizes the image into different groups and detects the object depending upon the contrast variation between groups. The Cascaded Haar classifier give us the set of potential locations for the vehicle presence.

Authors Name/s per Affiliation (Saad B. Alotaibi)

line 1 King Abdulaziz City for Science and Technology

line 2: Saudi Arabia, Riyadh

line 4: sbalotaibi@kacst.edu.sa

Authors Name/s per Affiliation (Amer S. Alharthi)

line 1 King Abdulaziz City for Science and Technology

line 2: Saudi Arabia, Riyadh

line 4: sbalotaibi@kacst.edu.sa

\section{RELATED WORK}

Most of recent works on moving object detection faced some of challenges as high performance and accurate approach. One of the recent related work in moving object detection, the researchers combined two algorithms namely, Ada boost cascade algorithm of Haar features and pyramidal KLT tracker algorithm. The Ada boost algorithm as presented by Viola Jones was limitedly used for object detection purpose. This algorithm uses a particular set of parameters to detect an object. The numbers of parameters are limitedly used in the presented case. The reason for the limited used was to reduce the computational process thereby increasing the identification speed. The training process was carried out with a minimum number of parameters as required for identification of a specific object. The identification was further speeded up and made reliable by using pyramidal KLT algorithm. The KLT algorithm utilizes the spatial intensity related information to detect the object. In this work, the Ada boost algorithm detects the object with minimal salient points and the pyramidal KLT further re-calculates the feature of the object based on the salient points that are detected through Ada boost algorithm. A specific algorithm is used for combining these two algorithms. This process makes it quicker and more reliable. The purpose of using two algorithms is based on the nature of the image. The image (road with cars) as used in the paper has cluttered background and the error percentage of any particular algorithm may be higher. By combining two algorithms the researchers have tried to ensure reliable and quick results[1]. While implementing the algorithm, the researchers have followed certain specific steps. Initially, certain minimally required tracking points such as edge features, line features, window boundaries and others required features of a car were identified and training process was completed. The training process included positive and negative samples to avoid true negative and false positive. The image was then read and the car detection process was carried out using the classifiers. For each of the detection certain salient features were extracted and stored. 
The salient features were further used to confirm the object's (car) presence by using the tracking algorithm. If the tracking algorithm confirms the object's presence then the presence of car is confirmed by the system and the car counter is increased by unity[1].

Other work has been carried out in two stages. The first stage involved a Haar classifier and the second stage used a thermal imagery to detect the presence of thermal signature. The combination of the two stages increased the confidence level with very few false positives. Haar classifier regionalises the image into multiple rectangles (groups) at a localised scale and tries to understand the contrast variation among the group. This information is used to detect the presence of a feature. Each individual classifier is capable of identifying a feature. Many such features are cascaded to increase the reliability and the confidence level. The features used for training the classifier must be optimally selected to get reliable results. In order to detect the human presence, multiple feature trained Haar classifiers were used. The confirmation for the detection was carried out through multivariate Gauss shaping technique. The mean-shift clustering process with edge detection was used to confirm the detection. The mean-shift clustering actually estimated the contours by determining the pixel variations in the thermal image. The car detection process also involved Haar classifiers as the primary detection source. Images with four different positional orientations at $00,450,900$ and 1350 with respect to the horizontal axis of the camera were used by the classifiers to detect the image. The secondary verification was done using the thermal imagery. The thermal hot spots as present in the thermal image were considered as evidence to detect the vehicle presence. The thermal hot spot points were analysed using watershed algorithm to generate the seed point. The seed point is the reference for the detection of the vehicles[2].

Vehicles come in different sizes, shape and colour. A traffic image at any instant may contain different types of vehicles with variant backgrounds. Real time detection of vehicles in such an image is all the more difficult and a complex task. The researchers overcome this challenging task through a two stage vehicle detection process. The first stage involves primary detections of the vehicle and the second stage involves secondary verification of the detected object and confirmation. In the first stage, Haar classifiers have been employed to detect vehicle. Haar classifier is a popular technique for human face detection and it involves very less computational process, thereby supporting the real time applications. It involves cascading of multiple classifiers to form a Haar like feature. In the second stage, the results of the primary detections are verified against a set of pre designated rules and logics to confirm the presence of the vehicle. Cascaded Haar classifier gives the set of potential locations for the vehicle presence. A fuzzy logic based approach is followed in the verification process which utilizes the Haar classifier output and to ensure the presence of vehicle[3].

We implement a Cascade Haar algorithem to detection vehicles in order to extract the traffic jam in real-time Using UAV. The following section describe a Cascade Haar approach that we are selected.

\section{Cascade Haar classifier}

Cascade Haar classifier is one of the recognized algorithms for object detection in an image with minimal computer processing. This algorithm has a reasonable accuracy for detecting even dynamic objects (same object with multiple forms). The commonly available algorithm reads the pixel color and plays with its intensity values to detect objects. In contrary to common algorithms, Haar classifier regionalizes the image into different groups and detects the object depending upon the contrast variation between groups. The fundamental prerequisite for the Haar classifier is the formation of integral image. An integral image actually contains the intensity information of the pixels that are to the left and top of the reference image. The reference image can be grouped in multiple rectangles and integral image can be generated for each group to detect the required feature. Integral image can be estimated with ease and requires very less computation. A minimum of one Haar classifier (formation of integral image) is required to identify a particular feature. Many such classifiers can be cascaded to detect multiple features thereby detecting the object with minimum false positive. For example, in order to detect human face three classifiers can be cascaded one each for mouth, eyes and nose. The presence of these three features will confirm the detection of human face. Prior to using of Haar classifiers, the same must be trained for identifying the exact required feature. The training is performed with a set of negative images and positive images. Thousands of images are used to train the classifier about the presence or absence of the specific feature. Increasing the number of images may increase the reliability but also increase false alarm rate. The images used for training must have large variations among themselves for ensuring robust feature detection[4].

The performance of the Haar classifiers can be further increased by regionalizing. By doing so, the region of image being used for feature detection is reduced and the number of false positives will reduce drastically. Regionalizing also reduces the computation processing and increase accuracy. Haar classifier is a simple and effective algorithm for object detection in an image. The feature selection and classifier training must be carefully chosen for increasing the positive hit. In the following section we will describe how to implement the Cascade Haar classifiers[4].

\section{v. Experiments and Results}

The UAV used for the experiment was could carry a weight of $1.2 \mathrm{~kg}$ (Gopro Camera, Transmitter and batteries) at an altitude of $20 \mathrm{~m}$. It carried an Gopro camera and was operated using the autopilot. The camera was positioned at $45^{\circ}$ to the horizontal axis of the flight direction. The UAV was controlled by Autopilot through GPS waypoints over IP radio link. The results were iterated and the experiments were carried out at different environmental conditions to generate the output figures. The results obtained through the experiments could satisfactorily detect the vehicles in an image but was not $100 \%$ perfect. A vehicle that was 
undetected in one of the image had every possibility to get detected in another one. The false positive thereby reduced to a large extent. The results showed some error on account of the camera angle, GPS error, wind conditions, altitude error and other varying environment conditions. The following figures show the results of real-time traffic detection (cf. figure3-7).
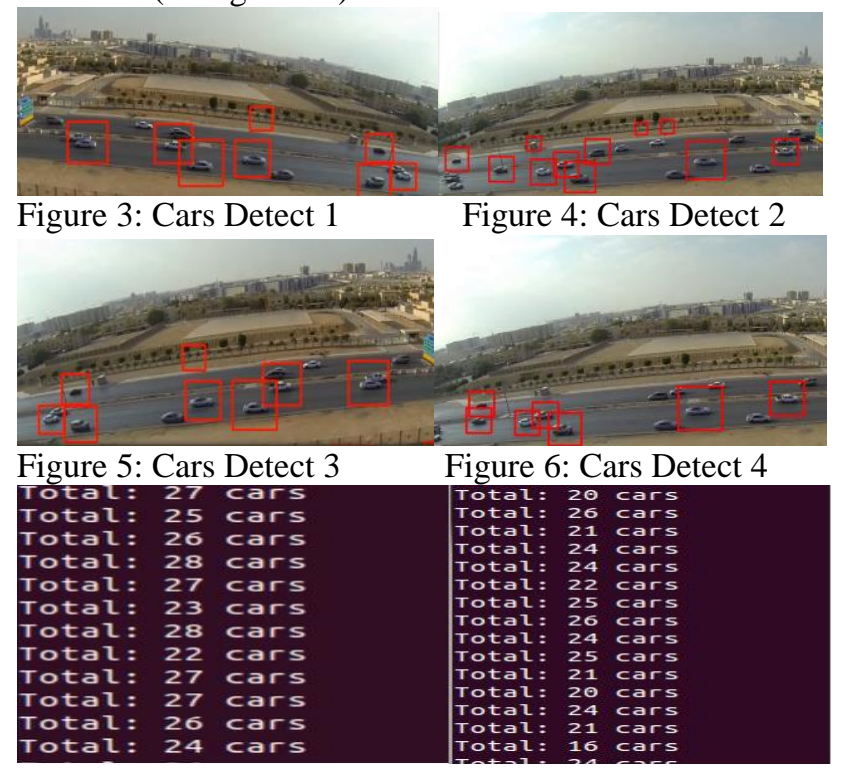

Figure 7: Cars Counter

\section{vi. Conclusion and Future Work}

We have done an excellent work in the area of real time detection of a moving or static vehicle. Achieving any real time application is a real challenging task and has been achieved satisfactorily in this work. The algorithm as used in the paper is Haar Cascade. This algorithm is used for object detection and are widely accepted for its quick and reliable action. As we say in future work, we will use ROS to control multi UAVs at different waypoints and make a analyzing and processing of traffic detection in the UAVs.

\section{References}

[1] Chirag I. Patel and Ripal Patel, "Counting Cars in Traffic Using Cascade Haar with KLP," International Journal of Computer and Electrical Engineering vol. 5, no. 4, pp. 435-437, 2013.

[2] A. Gszczak, T. P. Breckon, and J. Han, "Real-time people and vehicle detection from UAV imagery," in Intelligent Robots and Computer Vision: Algorithms and Techniques, Proceedings of SPIE, 2011.

[3] T. P. Breckon, S. E. Barnes, M. L. Eichner, and K. Wahren, "Autonomous real-time vehicle detection from a medium-level UAV," in Proceedings of the 24th International Conference on Unmanned Air Vehicle Systems, 2009.

[4] P. Wilson and J. Fernandez, "Facial feature detection using haar classifiers," The Journal of Computing Sciences in Colleges, vol. 21, pp. 127-133, 2006. 